\title{
Two Degree Becker Model for Mixture Design: Using D-optimal and A-optimal with Qualitative Factor
}

\author{
Zahra Rasooli Berardehi' and Chongqi Zhang ${ }^{2 *}$ \\ 'School of Mathematics and information Sciences, Guangzhou University, Guangzhou, China; \\ z.rasooli@e.gzhu.edu.cn \\ ${ }^{2}$ School of Statistics and Economics, Guangzhou University, Guangzhou, China; edu.cncqzhang@gzhu.edu.cn
}

\begin{abstract}
Objectives: To achieve an optimal approximation for two-degree Becker model in mixture design. Methods/Statistical Analysis: The problem of mixture design case, based on qualitative factors and finding A-optimal and D-optimal design for two-degree Becker model is investigated. With the aim of this issue, a generalization of Lee method is utilized. We proposed a new procedure of Lee method for approximation of Becker model. Moreover, simulation results are done in R software. Findings: There is a direct relation between qualitative factor and A-optimal and D-optimal design, such that, on the region of factors, if the qualitative factors have a uniform design then the trace of the inverse of information matrix is minimize for A-optimal design; and maximization of the determination of information matrix is essential for D-optimal design. Besides, for a product function, based on 3 sections corresponding to the 2-marginal design, the dispersion function can be detected. In addition, illustrated examples confirm the analytical results. Application/Improvements: The application of this work is to be used in engineering and manufacturing which need to an amount of convenient mixture design.
\end{abstract}

Keywords: Becker Model, Dispersion Function, Information Matrix, Mixture Experiment, Optimality

\section{Introduction}

Mixture experiment is one of the main procedures of manufactoring of a product and it has a vast range of application in industrial and technology. For instance, in Civil engineering ${ }^{1}$ Chemical sciences ${ }^{2}$, medicine ${ }^{\underline{3}}$ and so on, one can see the role of mixture design in advance ${ }^{4,5}$. There are many forms of dietary supplements, for example, tablets, capsules, liquids, powders, and gels. Dietary supplements are different from drugs, and they are nonpatent drugs. The Food and Drug Administration (FDA) defined a dietary supplement as an alternative food containing essential nutrients like vitamins, minerals, and proteins ${ }^{6}$. Subsequently, the Nutrition Labeling and Education Act of 1990 added herb or nutritional substances to the definition. In the pharmaceutical industry, tablets are the most acceptable form for consumers in comparison with other oral dosage forms ${ }^{\underline{7}}$. Tablet oral dosage has many advantages such as its ease of handling, chemical and physical stability, and portability. Furthermore, this type of dosage form ensures accuracy and consistency of dosages ${ }^{-8}$ There are many examinations that can be done in order to maintain the physical qualities of the tablets, for example, hardness test, percentage of friability test, disintegration test, and dissolution test ${ }^{9}$. Tablets are mixtures of active ingredients and other excipients. Mixtures mean the sum of all the ingredients is $100 \%$. There are many types of excipient with their own function in dosage formulation: diluents or fillers, binders, lubricants, glidants, antiadherents, disintegrates, colorants, and flavor or sweeteners. The mixture design statistical method is the most suitable method used in optimizing the tablet production process. The mixture design method is usually used in mixture formulation.

Here, there is the mean response at the $\mathrm{j}$-th level of a s-level for qualitative factor as follows

$$
E[y(j, \tau)]=f_{1}^{T}(\tau) \beta_{j}+f_{2}^{T}(\tau) \gamma, \quad \tau \in \chi \quad 1
$$


where, $f_{1}^{T}(\tau)$ denote the part of the regression functions having interaction with the qualitative factor and $f_{1}^{T}(\tau)$ can be seen as the part of the effect of the level, but $f_{2}^{T}(\tau)$ is the part which is invariant at each qualitative level and $f_{2}^{T}(\tau) \gamma$ can be mention as the sector of common effect. Also, $\beta_{j}=\left(\beta_{j 1}, \beta_{j 2}, \ldots, \beta_{\dot{p}_{1}}\right)^{T}, j=1,2, \ldots, s$ and $\gamma=\left(\gamma_{1}, \gamma_{2}, \ldots, \gamma_{p_{2}}\right)^{T}$ are vectors of unknown parameters, respectively. The experimental region of quantitative factors $x$ is the $q$ component mixture system which can be expressed as Where the $C^{\prime}{ }^{\prime}$ shows additional constraints condition which is introduced in 10 . Also, $f_{1}(\tau)$ and $f_{2}(\tau)$, as two parts of regression function, are $p_{1}-$ and $p_{2}-$ dimension vector including the quantitative effects, respectively. It is clear that, the model 1 is more effective to fixing and demonstrate the relationship between variables.

The fundamental objective of this study is to develop the results of the work $\mathrm{k}^{11}$ to the A-optimal designs of mixture model and $\frac{12,13}{}$ the $\mathrm{D}$-optimal deign of mixture model. The rest of the artice is arranged as follows: In section 2, some basic preliminaries and some notations are provided. Also, calculation of the trace of information matrix of model (1) is provided therein. In addition, the main results are given in section 3. And we find the A-optimal design and D-optimal design for the wo degree Becker model based on different situations of model (1). Finally, section 4 provides concluding remarks.

\section{Preliminaries}

The general linear model given by $E[y(z)]=g^{T}(z) \theta$ , where $y(z)$ is the response variable, $\theta$ is a vector of unknown parameters, $g(z)$ is a given vector of regression functions of $z \in \Omega$. An approximate design is a probability distribution with finite support on the factor space $\Omega$ and it is represented by $\zeta=\left(z_{1}, z_{2}, \ldots, z_{n} ; w_{1}, w_{2}, \ldots w_{n}\right) \quad$ which assigns, respectively. Masses $w_{1}, w_{2}, \ldots, w_{n} ; w_{i}>0, \sum w_{i}=1$ , to the $n$ distinct support points of $z_{1}, z_{2}, \ldots, z_{n}$ the design $s$ in the experimental region. The worth of a design is measured by its Fisher information matrix which is given by

$$
M(\zeta)=\int_{\Omega} g(z) g^{T}(z) \zeta(d z) .
$$

\subsection{A-optimal}

A design is defined to be A-optimal if it minimizes the trance of the inverse of the information matrix. The works $s^{14,15}$ gave us an effective way to check the A-optimality of arbitrary designs $\zeta$, and for a design $\zeta$ which is A-optimal if and only if

$$
g^{T}(z) M^{-2}(\zeta) g(z)-t\left[M^{-1}(\zeta)\right] \leq 0
$$

Let the general mode (1) be rewritten as

$$
E[y(j, \tau)]=\left[e_{j}^{T} \otimes f_{1}^{T}(x), f_{2}^{T}\right]\left(\beta_{1}^{T}, \beta_{2}^{T}, \ldots, \beta_{s}^{T}, \gamma^{T}\right)=g^{T}(j, \tau) \theta
$$

where, $e_{j} \in R^{s}$ is the unit vector whose $\mathrm{j}$-th component is equal to 1 and all others are 0 and $\otimes$ is used to denote the Kronecker product of two matrices, let $x_{s}=\{1,2, \ldots, s\}$ be the index set of the qualitative levels and $\Omega=x_{s} \times x$ be the experimental region. Note the information matrix of the design $\xi$ is:

$$
M_{f}(\xi)=\left[\begin{array}{ll}
M_{11}(\xi) & M_{12}(\xi) \\
M_{21}(\xi) & M_{22}(\xi)
\end{array}\right]
$$

which is associated with the model $E[y(\tau)]=\left[f_{1}^{T}(\tau), f_{2}^{T}(\tau)\right]\left(\beta^{T}, \gamma^{T}\right)^{T} . \quad$ An $\quad$ arbitrary design on $\Omega$ can be expressed as

$$
\zeta(j, \tau)=\eta(j) \xi(\tau)
$$

where, $\eta$ and $\xi_{j}$ are the marginal and the conditional designs on $\chi_{s}$ and $\chi$, respectively. 
If $\zeta$ is supposed as a design production and it is presented by $\zeta=\eta \times \xi$, where emphasizes that $\xi_{j}=\xi$ for all $j$.

According to the result of ${ }^{11}$ the information matrix of $\zeta$ will present by

$$
M_{g}(\zeta)=\left[\begin{array}{cc}
D \otimes M_{1}(\xi) & \eta \otimes M_{\mathfrak{2}}(\xi) \\
\eta^{T} \otimes M_{2}(\xi) & M_{2}(\xi)
\end{array}\right]
$$

In which

$$
\begin{aligned}
& M_{u v}(\xi)=\int_{x} f_{u}(\tau) f_{v}^{T}(\tau) \xi(d \tau), u, v \in\{1,2\}, \text { and } \\
& D=\operatorname{diag}(\eta(1), \eta(2), K, \eta(s)), \eta=(\eta(1), \eta(2), K, \eta(s))^{T}
\end{aligned}
$$

calculate the inverse matrix of $M_{g}(\varsigma)$. Now, the following lemma can be obtained.

Lemma $1 \frac{16,17}{}$ For, an arbitrary design $\zeta(j, \tau)=\eta(j) \times \xi(\tau)$ where $\eta$ and $\xi$ there are the conditional designs and the marginal on $x_{s}$ and $x$, respectively. Then one can has the following equation of trace for model (1).

$$
\operatorname{tr}\left[M_{g}^{-1}(\zeta)\right]=\operatorname{tr}\left(M_{11}^{-1}(\xi)\right) \sum_{j=1}^{s} \frac{1}{\eta(j)}+\operatorname{s.tr}\left(K_{(1)}\right)+\operatorname{tr}\left(D_{22}(\xi)\right)
$$

where,

$$
D_{22}(\xi)=\left[M_{22}(\xi)-M_{21}(\xi) M_{11}^{-1}(\xi) M_{12}(\xi)\right]^{-1}
$$

and

$$
K_{(1)}=M_{11}^{-1}(\xi) M_{12}(\xi) D_{22}(\xi) M_{21}(\xi) M_{11}^{-1}(\xi)
$$

\section{Proof:}

By calculating the inverse matrices of $M_{f}(\xi)$ and $M_{g}(\varsigma)$, we have

$$
\begin{gathered}
M_{f}^{-1}(\xi)=\left[\begin{array}{cc}
M_{11}^{-1}(\xi)+K_{(1)} & -M_{11}^{-1}(\xi) M_{12}(\xi) D_{22}(\xi) \\
-D_{22}(\xi) M_{21}(\xi) M_{11}^{-1}(\xi) & D_{22}(\xi)
\end{array}\right] \\
M_{g}^{-1}(\zeta)=\left[\begin{array}{ll}
D_{11}(\zeta) & D_{12}(\zeta) \\
D_{21}(\zeta) & D_{12}(\zeta)
\end{array}\right]
\end{gathered}
$$

where, $1_{S}$ is $S \times 1$ vector of all ones.

$$
\begin{gathered}
D_{\mathbb{1}}(\zeta)=D^{-1} \otimes M_{1}(\xi)+J_{s} \otimes K_{(1)}, \\
D_{12}(\zeta)=-1_{s} \otimes\left[M_{11}^{-1}(\xi) M_{12}(\xi) D_{22}(\xi)\right]=D_{21}^{T}(\zeta), \\
D_{22}(\zeta)=\left[M_{22}(\xi)-M_{21}(\xi) M_{11}^{-1}(\xi) M_{12}(\xi)\right]^{-1}=D_{22}(\xi), \\
\quad \text { and } \quad K_{(1)}=M_{11}^{-1}(\xi) M_{12}(\xi) D_{22} M_{21}(\xi) M_{11}^{-1}(\xi) .
\end{gathered}
$$

So we have

$$
\begin{aligned}
& \operatorname{tr}\left[M_{g}^{-1}(\zeta)\right]=\operatorname{tr}\left[D^{-1} \otimes M_{11}^{-1}(\xi)\right]+\operatorname{tr}\left[J_{s} \otimes K_{(1)}\right]+\operatorname{tr}\left[D_{22}(\xi)\right] \\
& =\operatorname{tr}\left(M_{11}^{-1}(\xi)\right) \sum_{j=1}^{s} \frac{1}{\eta(j)}+s \cdot \operatorname{tr}\left(K_{(1)}\right)+\operatorname{tr}\left(D_{22}(\xi)\right)
\end{aligned}
$$

And this completes the proof.

In particular, while the design $\eta(j)$ is a uniform design on $x_{s}$, i.e. $\eta(j)=\frac{1}{s}, j=1,2 . K, s$, then we have

$$
\begin{aligned}
& \operatorname{tr}\left[M_{g}^{-1}(\zeta)\right]=s^{2} \cdot\left(M_{11}^{-1}(\xi)\right)+s \cdot \operatorname{tr}\left(K_{(1)}\right)+\operatorname{tr}\left(D_{22}(\xi)\right) \\
& =s^{2} \cdot\left(M_{11}^{-1}(\xi)\right)+\left(s^{2}-s\right) \cdot \operatorname{tr}\left(M_{11}^{-1}(\xi)\right)+(1-s) \cdot \operatorname{tr}\left(D_{22}(\xi)\right)
\end{aligned}
$$

Moreover, it also shows that, if $\varsigma$ be an A-optimal, then all of the elements of $\eta$ should be equal, i.e.

$$
\eta(j)=\frac{1}{s}, \quad j=1,2, K, s .
$$

\subsection{D-optimal}

In a design, if the determine of information matrix be maximizes the with that design, then it is as a D-optimal design. A useful way for checking the D-optimality is attention to this point that a design can be $\mathrm{D}$-optimal if and only if

$$
g^{T}(j, \tau) M_{g}^{-1}(\xi) g^{T}(j, \tau)-s p_{1}-p_{2} \leq 0
$$

where, the information matrix of the design is

$$
M_{f}(\xi)=\left[\begin{array}{ll}
M_{11}(\xi) & M_{12}(\xi) \\
M_{21}(\xi) & M_{22}(\xi)
\end{array}\right]
$$

which, it is shown in (3) and this is same as information matrix with A-optimal design. 
In next section we will consider finding of the A-optimal and A-optimal designs for two degree Becker model under the this condition which:

$\eta(j)=\frac{1}{s}, \quad j=1,2, K, s$.

\section{Methodology}

In this part, A-optimal and D-optimal method for the two degree Becker model are investigated.

\subsection{A-optimal for the Two Degree Becker}

\section{Model}

For proving the A-optimality via the equivalence theorem, we can define the function as

$$
\Psi_{g}(z ; \zeta)=g^{T}(z) M_{g}^{-2}(\zeta) g(z), \quad z \in \Omega
$$

Based on the equivalence condition (2), and for any design $\zeta$, this is an A-optimal design if and only if following condition satisfy

$$
\Psi_{g}(z ; \zeta)-t\left[M_{g}^{-1}(\zeta)\right] \leq 0
$$

Theorem 2 Suppose that all conditions of Lemma 1 be confirmed and consider $\eta(j)=1 / s, \quad j=1,2, K, s$, then we have

$$
\begin{gathered}
\Psi_{g}(j, \tau ; \zeta)=s . \Psi_{f}(\tau ; \xi)+(1-s) \\
\|z(\tau ; \xi)\|^{2}+\left(s^{2}-s\right) \Psi_{f_{1}}(\tau ; \xi),
\end{gathered}
$$

and

$$
z(\tau ; \xi)=\left[-D_{22}(\xi) M_{21}(\xi) M_{11}^{-1}(\xi), D_{22}(\xi)\right] f(\tau), f^{T}(\tau)=\left[f_{1}^{T}(\tau), f_{2}^{T}(\tau)\right] .
$$

\section{Proof:}

For the convenience, we simply write $(0.3)$ as $M_{f}(\xi)=\left\{M_{j}\right\}_{i, j=1}^{2}$, then the following results can be obtained.

$$
\begin{aligned}
& \Psi_{g}(j, \tau ; \xi)=\frac{1}{\eta(j)} \Psi_{f}(\tau ; \xi)+\left(s-\frac{1}{\eta(j)}\right)\left[\sum_{i=1}^{4} A_{i}(\tau ; \xi)\right] \\
& +\left(1-\frac{1}{\eta(j)}\right)\left[\sum_{i=1}^{4} B_{i}(\tau ; \xi)\right] \\
& \quad+\left(\frac{1}{\eta^{2}(j)}-\frac{1}{\eta(j)}\right) \Psi_{f_{1}}(\tau ; \xi)
\end{aligned}
$$

where,

$$
\begin{aligned}
& A_{1}(\tau ; \xi)=f_{1}^{T}(\tau) K_{(1)}^{2} f_{1}(\tau), A_{2}(\tau ; \xi)=f_{1}^{T}(\tau) K_{(1)} M_{1}^{-1} M_{2} D_{2} f_{2}(\tau), \\
& A_{3}(\tau ; \xi)=f_{2}^{T}(\tau) D_{2} M_{2} M_{1}^{-1} K_{(1)} f_{1}(\tau), \\
& A_{4}(\tau ; \xi)=f_{2}^{T}(\tau) D_{2} M_{2} M_{1}^{-1} M_{\mathrm{L}} D_{2} f_{2}(\tau) . \\
& B_{1}(\tau ; \xi)=f_{1}^{T}(\tau) K_{(2)} f_{1}(\tau), B_{2}(\tau ; \xi)=f_{1}^{T}(\tau) M_{1}^{-1} M_{\mathrm{L}} D_{2}^{2} f_{2}(\tau), \\
& B_{3}(\tau ; \xi)=f_{2}^{T}(\tau) D_{2}^{2} M_{\mathbf{2}} M_{1}^{-1} f_{1}(\tau), \\
& B_{4}(\tau ; \xi)=f_{2}^{T}(\tau) D_{2}^{2} f_{2}(\tau) . \\
& \quad \text { and } K_{(2)}=M_{1}^{-1} M_{\mathrm{L}} D_{2}^{2} M_{2} M_{1}^{-1} . \text { Because } \\
& \sum_{i=1}^{4} B_{i}(\tau ; \xi)=\|z(\tau ; \xi)\|^{2}=\left\|\left[-D_{22}(\xi) M_{12}(\xi) M_{11}^{-1}(\xi), D_{22}(\xi)\right] f(\tau)\right\|^{2}
\end{aligned}
$$

It is obviously that the theorem is hold when $\eta(j)=1 / s, j=1,2, K, s$.

So, proof is finished.

Corollary 1 As a result of the lemma 1 and theorem 1, one has

$\psi_{g}(z, \tau ; \zeta)-\operatorname{tr}\left[M_{g}^{-1}(\zeta)\right]=s t_{1}(\tau ; \zeta)+\left(s^{2}-s\right) t_{2}(\tau ; \zeta)+(1-s) t_{3}(\tau ; \zeta)$

where,

$$
\begin{aligned}
& t_{1}(\tau ; \zeta)=\psi_{f}(\tau ; \zeta)-t\left[M_{g}^{-1}(\zeta)\right], \\
& t_{2}(\tau ; \zeta)=\psi_{f_{1}}(\tau ; \zeta)-t\left[M_{1}^{-1}(\zeta)\right], \\
& t_{3}(\tau ; \zeta)=\|z(\tau ; \zeta)\|^{2}(\tau ; \zeta)-t\left[D_{2}(\xi)\right],
\end{aligned}
$$

And then by considering of the $q$ components twodegree Becker model symbol as

$$
E[y(\tau)]=\sum_{k=1}^{q} f_{l_{k}}^{T}(\tau) \beta_{k}, \tau \in \chi \subseteq s^{q-1}
$$

where,

$$
\begin{aligned}
& f_{L_{1}}(\tau)=\left(x_{1}, x_{2}, \ldots, x_{q}\right)^{T} \\
& f_{L_{2}}(\tau)=\left(\sqrt{x_{1} x_{2}}, \sqrt{x_{2} x_{3}}, \ldots, \sqrt{x_{q-1} x_{q}}\right)^{T} \\
& f_{L_{1}}(\tau)=\left(x_{1} x_{2} x_{3}, x_{2} x_{3} x_{4}, \ldots, x_{q-2} x_{q-1} x_{q}\right)^{T}, \ldots, f_{L_{q}}(\tau)=x_{1} x_{2} x_{3} \ldots x_{q}
\end{aligned}
$$

Now, for fixing the ideas, we should focus on the model which is provided on $S^{q-1}$ by

$$
E\left[y(\tau)=f_{L_{1}}^{T}(\tau) \beta_{1}+f_{L_{2}}^{T}(\tau) \beta_{2} .\right.
$$


Three kinds of model which form as (1) given, must be considered. Means, for the general model of multiresponse

$$
E\left[y(j, \tau]=\left(f_{L_{1}}^{T}(\tau), f_{L_{2}}^{T}(\tau)\right) \beta_{j}, j=1,2, \ldots, s\right.
$$

where, on the different levels it has different function, and it doesn't have any qualitative factors.

If we assume the $f_{L_{2}}^{T}(\tau)$ be a qualitative factors and suppose that $f_{L_{1}}^{T}(\tau)$ having interaction with the qualitative factor, then this model can be presented as

$$
E\left[y(j, \tau]=f_{L_{1}}^{T}(\tau) \beta^{\left(L_{1}\right)}{ }_{j}+f_{L_{2}}^{T}(\tau) \gamma^{\left(L_{2}\right)}\right.
$$

Equivalently, the two part of regression function can be exchanged as quantitative and qualitative factors, so the model change as

$$
E\left[y(j, \tau]=f_{L_{2}}^{T}(\tau) \beta^{\left(L_{2}\right)}+f_{L_{1}}^{T}(\tau) \gamma^{\left(L_{1}\right)}\right.
$$

However, there isn't any main difference between model (6) to model (8). In this study, qualitative and quantitative factors are considered altogether, the problem of design to estimate the unknown parameters will be supposed where it is considered to exist one qualitative factor with $s$ levels. The two degree Becker model mentioned that for models (6),(7) and (8), $\psi_{g}(j, \tau ; \zeta)$ gains its maximum just at the barycentres of $S^{q-1}$. Therefore, just the barycentres can be possible in the support points for designs of $A$-optimal.

In first step, define $M_{i}$ as a $C(q, i) \times q$ matrix, such that the first $i$ elements in the first row of $M_{i}$ are 1 and the other elements in the first row equal to 0 , and the other $C(q, i)-1$ rows of $M_{i}$ have the different permutations of the first row based on lexicographical order. (For instance, in the case of $i=2$ and $q=4, M_{i}$ is a $6 \times 4$ matrix, and its 1 st, 2 nd, $\cdots$, 6 th rows should be $(1,1,0,0)(1,0,1,0)(1,0,0,1)(0,1,1,0)(0,1,0,1)(0,0,1$,$) ,$ respectively.)

Consider that $T_{i}$ be definition of the points set which elements are each rows of $i^{-1} M_{i}, i=1,2, \ldots, q$. Then, the $T_{1}$ called the set of all vertexes of $S^{q-1}, T_{2}$ called the set of barycenter on the $q-2$ ension boundary. So, the design $\xi$ can be define according to the models (6),(7) and (8) as following equation:

$$
\xi=\left(T_{1}, T_{2} ; w_{1}, w_{2}\right)
$$

where, the weight functions $w_{1}$ and $w_{2}$ satisfy the condition $q w_{1}+C(q, 2) w_{2}=1$.

Therefore,for the information matrix $M_{f}(\xi)$ which is associated via model (6), one can has

$$
M_{f}(\xi)=\left[\begin{array}{cc}
\frac{w_{2}}{4} M_{2}^{T} M_{2} I_{Q}+w_{2} I_{Q} & \frac{w_{2}}{2} M_{2}^{T} M_{2} I_{Q} \\
\frac{w_{2}}{4} M_{2} I_{Q} & \frac{w_{2}}{2} I_{Q}
\end{array}\right]
$$

where, $Q=q(q-1) / 2$, and $I_{q}$ is the $q \times q$ identity matrix. According to these notations, the following Lemma can be expressed.

Lemma 2 For any design $\xi$ such as (9) defined, the function $\psi_{g}(j, \tau ; \xi)$ with $f^{T}(\tau)=\left\lfloor f_{1}^{T}(\tau)+f_{2}^{T}(\tau)\right\rfloor$ in one of the (7) and (8) models can be displayed as

$$
\begin{aligned}
& \psi_{f}(\tau ; \xi)=a_{0}+\sum_{i=1}^{q}\left[a_{1} x_{i}^{2}+a_{3} x_{i}^{2}\left(1-x_{i}\right)+a_{6} x_{i}^{2}\left(1-x_{i}\right)^{2}\right] \\
& +\sum_{i<j}^{q}\left[a_{2} x_{i} x_{j}+a_{4} x_{i}^{2} x_{j}^{2}\right]+a_{5}\left(\sum_{i<j}^{q} x_{i} x_{j}\right)^{2}
\end{aligned}
$$

where,

$$
\begin{aligned}
& a_{0}=\frac{4}{w_{1}^{2}}, a_{1}=\frac{4 q-7}{w_{1}^{2}}, a_{2}=-\frac{32}{w_{1}^{2}}, a_{3}=\frac{64 w_{1}+4(4 q-7) w_{2}}{w_{1}^{2} w_{2}}, \\
& a_{4}=\frac{256}{w_{2}^{2}}, a_{5}=\frac{64}{w_{2}^{2}}, a_{6}=\frac{128 w_{1}+4(4 q-7) w_{2}}{w_{1}^{2} w_{2}}
\end{aligned}
$$

Also, in the model (7),

$$
\psi_{f_{1}}(\tau ; \xi)=\frac{16 q^{2}}{\left(q^{2} w_{2}-4\right)^{2}} \sum_{i=1}^{q} x_{i}^{2}+\frac{-8 q^{2} w_{2}+q^{5} w_{2}}{\left(q^{2} w_{2}-4\right)^{2}},\|z(\tau ; \xi)\|^{2}=\sum_{i<j}^{q} \delta_{i j}^{2}(\tau),
$$

which

$$
\begin{aligned}
& \delta_{i j}(\tau)=\left(\frac{4}{w_{1}}-c\right)\left(x_{i}+x_{j}\right)-\frac{4}{w_{1}}\left(x_{i}^{2}+x_{j}^{2}\right) \\
& +\frac{16}{w_{1}}\left(x_{i} x_{j}\right), \quad 1 \leq j \leq q
\end{aligned}
$$


and $c=\frac{4 q}{2+q w_{2}-q^{2} w_{2}}$.

Moreover, in the model (8),

$\psi_{f_{1}}(\tau ; \xi)=\frac{256}{w_{2}^{2}} \sum_{i<j}^{q} x_{i}^{2} x_{j}^{2},\|z(\tau ; \xi)\|^{2}=\sum_{i=1}^{q} \delta_{i}^{2}(\tau)$

which, $\delta_{i}(\tau)=-\frac{2}{w_{1}}\left(2 x_{i}^{2}-x_{i}\right), i=1,2, \ldots, q$.

Proof:

By calculating the inverse matrix of $M_{f}(\xi)$ is

$M_{f}^{-1}(\xi)=\left[\begin{array}{cc}\frac{1}{w_{1}} I_{q} & -\frac{2}{w_{2}} M_{2}^{T} \\ -\frac{2}{w_{1}} M_{2} & \frac{4}{w_{1}} M_{2} M_{2}^{T}+\frac{16}{w_{2}} I_{Q}\end{array}\right]$

Note $J(k, l)=1_{k} 1_{l}^{T}, J_{k}=1_{k} 1_{l}^{T}$ and the matrix $M_{f}^{-2}(\xi)=\left\{A_{j}\right\}_{i, j=1}^{2}$, where

$A_{11}=\frac{4 q-7}{w_{1}^{2}} I_{q}+\frac{4}{w_{1}^{2}} J_{q}, A_{12}=-\frac{16}{w_{1}^{2}} J(q, Q)-\frac{32 w_{1}+2(4 q-7) w_{2}}{w_{1}^{2} w_{2}} M_{2}^{T}=A_{21}^{T}$, $A_{22}=\frac{256}{w_{2}^{2}} I_{Q}+\frac{64}{w_{1}^{2}} J_{Q}+\frac{128 w_{1}+4(4 q-7) w_{2}}{w_{1}^{2} w_{2}} M_{2} M_{2}^{T}$.

For the model (0.6), the form of information matrix is same as (0.9), then we have

$$
\begin{aligned}
& M_{11}^{-1}(\xi)=-\frac{4 q}{4-q^{2} w_{2}} I_{q}+\frac{q^{2} w_{2}}{4-q^{2} w_{2}} J_{q}, \\
& M_{11}^{-2}(\xi)=\frac{16 q^{2}}{\left(q^{2} w_{2}-4\right)^{2}} I_{q}+\frac{-8 q^{3} w_{2}+q^{5} w_{2}^{2}}{\left(q^{2} w_{2}-4\right)^{2}} J_{q}, \\
& M_{11}^{-1}(\xi) M_{12}(\xi) D_{22}(\xi)=c M_{2}^{T},
\end{aligned}
$$

where, $c=\frac{4 q}{2+q w_{2}-q^{2} w_{2}}$. For the model (0.7),

note $\bar{M}^{-1}(\xi)=\left\{\bar{D}_{i j}(\xi)\right\}_{i, j=1}^{2}$,

the $f^{T}(x)=\left[f_{1}^{T}(x), f_{2}^{T}(x)\right]=\left[f_{L_{2}}^{T}(x), f_{L_{1}}^{T}(x)\right]$, the form of information matrix is

$$
\bar{M}_{f}(\xi)=\left\{\bar{M}_{i j}(\xi)\right\}_{i, j=1}^{2}=\left[\begin{array}{ll}
M_{22}(\xi) & M_{21}(\xi) \\
M_{11}(\xi) & M_{11}(\xi)
\end{array}\right]
$$

$$
\bar{M}_{11}^{-2}(\xi)=\frac{256}{w_{2}^{2}} I_{Q}, \bar{D}_{22}(\xi)=\frac{1}{w_{1}} I_{q}, \bar{M}_{11}^{-1}(\xi) \bar{M}_{12}(\xi) \bar{D}_{22}(\xi)=-\frac{16}{w_{2}} M_{2} .
$$

So we can obtain the result of $\psi_{f}(\tau ; \xi) \psi_{f_{1}}(\tau ; \xi)$ and $z(\tau ; \xi)$ by calculating $\quad f^{T}(x) M_{f}^{-2}(\xi) f(x) f_{1}^{T}(x) M_{1}^{-2}(\xi) f_{1}(x)$ and $\left\lfloor D_{2} M_{2} M_{1}^{-1}, D_{2}\right\rfloor f(x)$, respectively.

Then, the proof is completed.

Now, based on the condition $w_{1}=1 / q-w_{2}(q-1) / 2$ , Theorem 1 and Lemma 2, consider that $\tau_{i} \in T_{i}=1,2$, then the function (5) can be precised as

$h_{i}\left(w_{2}\right)=\psi_{g}\left(j, \tau_{i} ; \zeta\right)=s h_{i 1}\left(w_{2}\right)+\left(s^{2}-s\right) h_{i 2}\left(w_{2}\right)+(1-s) h_{i 3}\left(w_{2}\right)$.

Plus, in the model (7), we get

$$
\begin{aligned}
& h_{11}\left(w_{2}\right)=a_{0}+a_{1}, h_{12}\left(w_{2}\right)=\frac{16 q^{2}-8 q^{2} w_{2}+5 q^{5} w_{2}^{2}}{\left(q^{2} w_{2}-4\right)^{2}}, h_{13}\left(w_{2}\right)=(q-1) c^{2} ; \\
& h_{21}\left(w_{2}\right)=a_{0}+\frac{a_{1}}{2}+\frac{a_{2}}{4}+\frac{a_{3}}{4}+\frac{a_{4}}{16}+\frac{a_{5}}{16}+\frac{a_{6}}{8}, h_{22}\left(w_{2}\right)=\frac{8 q^{2}-8 q^{2} w_{2}+5 q^{5} w_{2}^{2}}{\left(q^{2} w_{2}-4\right)^{2}}, \\
& h_{23}\left(w_{2}\right)=\left(\frac{4}{w_{1}}+\frac{4}{w_{2}}-c\right)^{2}+(2 q-4)\left(\frac{4}{w_{1}}-\frac{c}{2}\right)^{2} .
\end{aligned}
$$

and in the model (8), $h_{\mathbb{1}}\left(w_{2}\right)$ and $h_{\mathrm{d}}\left(w_{2}\right)$ are same as model (7), then one can has $h_{12}\left(w_{2}\right)=0, h_{13}\left(w_{2}\right)=1 / w_{1}^{2} ; h_{22}\left(w_{2}\right)=16 / w_{2}^{2}, h_{23}\left(w_{2}\right)=0$.

Here, for finding of the $A$-optimal design $\zeta^{*}$ for the model (7) and (8), solving the following equation is needed.

$\psi_{g}\left(j, \tau_{1} ; \zeta^{*}\right)=\psi_{g}\left(j, \tau_{2} ; \zeta^{*}\right)$.

Clearly the solution of equation $w_{i}=u_{i}(q, s), i=1,2$ is too complicated to analysis and symbol, however, the approximate of the optimal design by calculate the result of Lemma 2 can be obtained. For instance, consider $q=30, s=20$, one can find the $A$-optimal design of $\zeta^{*}=\eta^{*} \times \xi^{*}$ on the region $X_{s} \times S^{q-1}$, which on $X_{s}$ region, the $\eta^{*}$ should be uniform design and we can obtain the optimal design $\xi^{*}$ on $S^{q-1}$ by calculat- 
ing of $\log h_{1}\left(w_{2}\right), \log h_{2}\left(w_{2}\right)$, and $\log \operatorname{tr}\left(M_{g}^{-1}(\zeta)\right)$ in $w_{2} \in(0,1 / Q)$.

Now, in model (7), one gets that $\min _{w_{2} \in(0,1 / Q)}\left\{\log t\left(M_{g}^{-1}(\zeta)\right\}=7.3761\right.$ for $w_{2}^{*}=0.0546$ , thus the design

$$
\xi^{*}=\left(T_{1}, T_{2} ; 0.0908,0.0546\right) .
$$

Also, in model (8), we obtain $\min _{w_{2} \in(0,1 / Q)}\left\{\log t\left(M_{g}^{-1}(\zeta)\right\}=7.0867 \quad\right.$ in which $w_{2}^{*}=0.0768$, thus the design

$$
\xi^{*}=\left(T_{1}, T_{2} ; 0.1348,0.0768\right) .
$$

the design of $\zeta^{*}$ can be verified as defined above implies equivalence condition (11) since the three curves $\log h_{1}\left(w_{2}\right) \log h_{2}\left(w_{2}\right)$ and $\log t\left(M_{g}^{-1}(\zeta)\right.$ intercross at the same point, where these are shown in Figures 1 and 2.
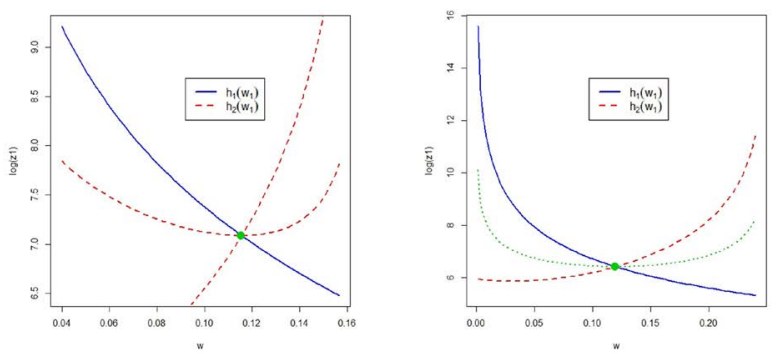

Figure 1. $A-$ optimal design on model (7) and model(8).
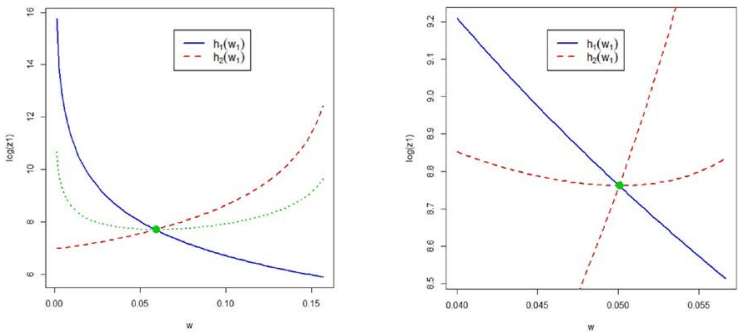

Figure 2. $D$ - optimal design on model (7) and model(8).
Here, the optimal weights for model (6), (7) and (8) are also listed with $q \in\{3,4, L, 6\}$ and $s \in\{2,3, L, 6\}$ as one can see in the Tables 1 and 2 .

Table 1. The weights of D-optimal design for

\begin{tabular}{|c|c|c|c|c|c|}
\hline & & \multicolumn{4}{|c|}{ Model (7) } \\
\hline$q$ & $S$ & $w_{1}$ & $w_{2}$ & $\min (y)$ & $\log \left(\operatorname{tr}\left(M_{g}\right)\right)$ \\
\hline 3 & 2 & 0.1735 & 0.1597 & 168.981 & 5.129 \\
\hline 4 & 2 & 0.1096 & 0.0935 & 485.255 & 6.184 \\
\hline 5 & 2 & 0.0773 & 0.0613 & 1114.969 & 7.0165 \\
\hline 6 & 2 & 0.0591 & 0.0429 & 2215.092 & 7.703 \\
\hline 3 & 3 & 0.1898 & 0.1433 & 233.806 & 5.453 \\
\hline 4 & 3 & 0.1192 & 0.0871 & 611.133 & 6.4150 \\
\hline 5 & 3 & 0.0830 & 0.0584 & 1324.596 & 7.188 \\
\hline 6 & 3 & 0.0623 & 0.0417 & 2532.032 & 7.836 \\
\hline 3 & 4 & 0.2061 & 0.1272 & 318.530 & 5.763 \\
\hline 4 & 4 & 0.1289 & 0.0807 & 775.176 & 6.653 \\
\hline 5 & 4 & 0.0907 & 0.0546 & 1597.447 & 7.3761 \\
\hline 6 & 4 & 0.0670 & 0.0398 & 2944.431 & 7.9876 \\
\hline 3 & 5 & 0.2191 & 0.1141 & 422.407 & 6.0459 \\
\hline 4 & 5 & 0.1386 & 0.0742 & 975.466 & 6.8829 \\
\hline 5 & 5 & 0.0964 & 0.0517 & 1930.49 & 7.5655 \\
\hline 6 & 5 & 0.0717 & 0.0379 & 3448.009 & 8.1455 \\
\hline 3 & 6 & 0.2321 & 0.1011 & 545.037 & 6.3008 \\
\hline 4 & 6 & 0.1482 & 0.0678 & 1210.69 & 7.0989 \\
\hline 5 & 6 & 0.1040 & 0.0479 & 2321.26 & 7.7498 \\
\hline 6 & 6 & 0.0764 & 0.0360 & 4038.83 & 8.3037 \\
\hline
\end{tabular}
$3 \leq q \leq 6$ and $2 \leq s \leq 6$ for Model (7)

The implementation of designs in comparison of the $A$-optimal design for model $g(\tau)$ are measured by the $A$-efficiency which is given by

$$
A_{e f f}(\zeta)=\frac{t\left[M_{g}^{-1}\left(\zeta^{*}\right)\right]}{t\left[M_{g}^{-1}(\zeta)\right]}
$$

It is worth noting that $\zeta_{j}^{*}=\eta^{*} \times \xi_{j}^{*}, j=1,2,3$ are $A$-optimal design for model (6),(7) and (8), respectively. These designs are compared reciprocally with together for $q \in\{3,4, L, 6\}$ and $s \in\{2,3, L, 6\}$ and the $A$-efficiencies are shown in Tables 3 and 4 . 
Table 2. The weights of $\mathrm{D}$-optimal design for

$3 \leq q \leq 6$ and $2 \leq s \leq 6$ for Model (8)

\begin{tabular}{|c|c|c|c|c|c|}
\hline & & \multicolumn{4}{|c|}{ Model $(8)$} \\
\hline$q$ & $S$ & $w_{1}$ & $w_{2}$ & $\min (y)$ & $\log \left(\operatorname{tr}\left(M_{g}\right)\right)$ \\
\hline 3 & 2 & 0.2245 & 0.1187 & 350.00 & 5.857 \\
\hline 4 & 2 & 0.1154 & 0.0768 & 1195.99 & 7.086 \\
\hline 5 & 2 & 0.0728 & 0.0543 & 3025.01 & 8.014 \\
\hline 6 & 2 & 0.0501 & 0.0414 & 6383.98 & 8.761 \\
\hline 3 & 3 & 0.2317 & 0.1015 & 672.74 & 6.511 \\
\hline 4 & 3 & 0.1236 & 0.0644 & 2366.78 & 7.769 \\
\hline 5 & 3 & 0.0768 & 0.0462 & 6088.33 & 8.714 \\
\hline 6 & 3 & 0.0526 & 0.0351 & 12966.00 & 9.472 \\
\hline 3 & 4 & 0.2431 & 0.0901 & 1089.03 & 6.993 \\
\hline 4 & 4 & 0.1283 & 0.0542 & 3896.57 & 8.267 \\
\hline 5 & 4 & 0.0793 & 0.0412 & 10123.57 & 9.222 \\
\hline 6 & 4 & 0.0543 & 0.0308 & 21775.07 & 9.987 \\
\hline 3 & 5 & 0.2489 & 0.0844 & 1596.13 & 7.375 \\
\hline 4 & 5 & 0.1319 & 0.0521 & 5776.14 & 8.661 \\
\hline 5 & 5 & 0.0814 & 0.0371 & 15107.61 & 9.622 \\
\hline 6 & 5 & 0.0553 & 0.0283 & 32614.38 & 10.392 \\
\hline 3 & 6 & 0.2575 & 0.0758 & 2191.95 & 7.692 \\
\hline 4 & 6 & 0.0134 & 0.0485 & 7988.78 & 9.987 \\
\hline 5 & 6 & 0.0829 & 0.0341 & 21025.43 & 9.953 \\
\hline 6 & 6 & 0.0561 & 0.0262 & 45542.74 & 10.726 \\
\hline
\end{tabular}

Table 3. Comparisons of $A$-Optimal for $3 \leq q \leq 6$ and $2 \leq s \leq 6$

\begin{tabular}{|c|c|c|c|c|c|}
\hline & & \multicolumn{4}{|c|}{ Model (7) } \\
\hline$q$ & $s$ & $w_{1}$ & $w_{2}$ & $\min (y)$ & $\log \left(\operatorname{tr}\left(M_{g}\right)\right)$ \\
\hline 3 & 2 & 0.1735 & 0.1597 & 168.981 & 5.129 \\
\hline 4 & 2 & 0.1096 & 0.0935 & 485.255 & 6.184 \\
\hline 5 & 2 & 0.0773 & 0.0613 & 1114.969 & 7.016 \\
\hline 6 & 2 & 0.0591 & 0.0429 & 2215.092 & 7.703 \\
\hline 3 & 3 & 0.1898 & 0.1434 & 233.806 & 5.454 \\
\hline 4 & 3 & 0.1192 & 0.0871 & 611.133 & 6.415 \\
\hline 5 & 3 & 0.0830 & 0.0584 & 1324.596 & 7.188 \\
\hline 6 & 3 & 0.0623 & 0.0417 & 2532.032 & 7.836 \\
\hline 3 & 4 & 0.2061 & 0.1272 & 318.530 & 5.763 \\
\hline 4 & 4 & 0.1289 & 0.0807 & 775.176 & 6.653 \\
\hline 5 & 4 & 0.0907 & 0.0546 & 1597.447 & 7.376 \\
\hline 6 & 4 & 0.0670 & 0.0398 & 2944.431 & 7.987 \\
\hline 3 & 5 & 0.2191 & 0.1141 & 422.407 & 6.045 \\
\hline 4 & 5 & 0.1386 & 0.0742 & 975.466 & 6.882 \\
\hline 5 & 5 & 0.0964 & 0.0517 & 1930.49 & 7.565 \\
\hline 6 & 5 & 0.0717 & 0.0379 & 3448.00 & 8.145 \\
\hline 3 & 6 & 0.2321 & 0.1011 & 545.037 & 6.300 \\
\hline
\end{tabular}

\begin{tabular}{|l|l|l|l|l|l|}
\hline 4 & 6 & 0.1482 & 0.0678 & 1210.69 & 7.098 \\
\hline 5 & 6 & 0.1040 & 0.0479 & 2321.26 & 7.749 \\
\hline 6 & 6 & 0.0764 & 0.0360 & 4038.87 & 8.303 \\
\hline
\end{tabular}

Table 4. Comparisons of $A$-Optimal for $3 \leq q \leq 6$ and $2 \leq s \leq 6$

\begin{tabular}{|c|c|c|c|c|c|}
\hline & & \multicolumn{4}{|c|}{ Model $(8)$} \\
\hline$q$ & $S$ & $w_{1}$ & $w_{2}$ & $\min (y)$ & $\log \left(\operatorname{tr}\left(M_{g}\right)\right)$ \\
\hline 3 & 2 & 0.2145 & 0.1187 & 350.00 & 5.857 \\
\hline 4 & 2 & 0.1154 & 0.0768 & 1195.99 & 7.086 \\
\hline 5 & 2 & 0.0728 & 0.0543 & 3025.01 & 8.014 \\
\hline 6 & 2 & - & - & - & - \\
\hline 3 & 3 & 0.2317 & 0.1015 & 672.74 & 6.511 \\
\hline 4 & 3 & 0.1236 & 0.0644 & 2366.78 & 7.769 \\
\hline 5 & 3 & 0.0768 & 0.0462 & 6088.33 & 8.714 \\
\hline 6 & 3 & - & - & - & - \\
\hline 3 & 4 & 0.2431 & 0.0901 & 1089.03 & 6.993 \\
\hline 4 & 4 & 0.1283 & 0.0542 & 3896.57 & 8.267 \\
\hline 5 & 4 & 0.0793 & 0.0412 & 10123.57 & 9.222 \\
\hline 6 & 4 & - & - & - & - \\
\hline 3 & 5 & 0.2489 & 0.0844 & 1596.13 & 7.375 \\
\hline 4 & 5 & 0.1319 & 0.0521 & 5776.14 & 8.661 \\
\hline 5 & 5 & 0.0814 & 0.0371 & 15107.61 & 9.622 \\
\hline 6 & 5 & - & - & - & - \\
\hline 3 & 6 & 0.2575 & 0.0758 & 2191.95 & 7.692 \\
\hline 4 & 6 & 0.0134 & 0.0485 & 7998.745 & 8.987 \\
\hline 5 & 6 & 0.0829 & 0.0341 & 21025.43 & 9.953 \\
\hline 6 & 6 & - & - & - & - \\
\hline
\end{tabular}

\section{Conclusion}

This study investigates the problem of mixture design case because of efficacy of mixture design in procedure of industrial experiences. In this regard, based on qualitative factors and finding A-optimal and D-optimal design for two-degree Becker model, the condition of production of mixture design is taken into account. It is worth to mention that, there is a direct relation between qualitative factor and A-optimal and D-optimal design. Such that, firstly on the region of factors, if the qualitative factors have a uniform design then the trace of the inverse of information matrix is minimize for A-optimal design. Secondly, maximization of the determination of information matrix is essential for D-optimal design. In addition, for a product function, based on three sections corresponding to the two marginal design, the dispersion function can be detected. 


\section{References}

1. Jiao D, Shi C, Yuan Q, An X, Liu Y. Mixture design of concrete using simplex centroid design method. Cement and Concrete Composites. 2018; 89:76-88. https://doi. org/10.1016/j.cemconcomp.2018.03.001

2. Jonuzaj S, Gupta A, Adjiman CS. The design of optimal mixtures from atom groups using Generalized Disjunctive Programming. Computers and Chemical Engineering. 2018; 116:401-21. https://doi.org/10.1016/j.compchemeng.2018.01.016

3. Ferreira-Nunes R, Gratieri T, Gelfuso GM, Cunha-Filho M. Mixture design applied in compatibility studies of catechin and lipid compounds. Journal of Pharmaceutical and Biomedical Analysis. 2018; 149:612-17. https://doi. org/10.1016/j.jpba.2017.11.069. PMid:29202440

4. Bondari K. Mixture experiments and their applications in agricultural research. Statistics and Data Analysis; 2005. p. 1-8.

5. Amir IZ, Syafiq A, Sharon WXR. Mixture experiment on rheological properties of dark chocolate as influenced by cocoa butter substitution with xanthan gum/corn starch/ glycerin blends. International Food Research Journal. 2014; 21(5):1887-92.

6. Zen NIM, Gani SSA, Shamsudin R, Masoumi HRF. The use of D-optimal mixture design in optimizing development of Okara tablet formulation as a dietary supplement. The Scientific World Journal; 2015. p. 1-7.

7. Wen H, Park K. Oral controlled release formulation design and drug delivery. Theory to Practice. John Wiley and Sons; 2011. p. 1-30.

8. Gad SC. Pharmaceutical manufacturing handbook: Production and processes. John Wiley and Sons; 2008. https://doi.org/10.1002/9780470259818
9. Seitz JA, Flessland GM. Evaluation of the physical properties of compressed Tablets I. Tablet hardness and friability. Journal of Pharmaceutical Sciences. 1965; 54(9):1353-7. https://doi.org/10.1002/jps.2600540926. PMid:5881235

10. Liu Y, Liu MQ. Construction of uniform designs for mixture experiments with complex constraints. Communications in Statistics-theory and Methods. 2016; 45(8):2172-80. https://doi.org/10.1080/03610926.2013.875576

11. Lee CP, Huang MNL. D-optimal designs for second-order response surface models with qualitative factors. Journal of Data Science. 2011; 9:139-53.

12. Huang MNL, Lee CP, Chen RP, Klein T. Exact D-optimal designs for a second-order response surface model on a circle with qualitative factors. Computational Statistics and Data Analysis. 2010; 54(2):516-30. https://doi. org/10.1016/j.csda.2009.09.022

13. Yue RZ, Liu X, Chatterjee K. D-optimal designs for multiresponse linear models with a qualitative factor. Journal of Multivariate Analysis. 2014; 124:57-69. https://doi. org/10.1016/j.jmva.2013.10.011

14. Kiefer J. Optimal design: Variation in structure and performance under change of criterion. Biometrika. 1975; 62(2):277-88. https://doi.org/10.1093/biomet/62.2.277

15. Kiefer J. General equivalence theory for optimum designs (approximate theory). The annals of Statistics. 1974; 2(5):849-79. https://doi.org/10.1214/aos/1176342810

16. Li G, Zhang C. The pseudo component transformation design for experiment with mixture. Statistics and Probability Letters. 2017; 131:19-24. https://doi. org/10.1016/j.spl.2017.07.017

17. Li G, Zhang C. Random search algorithm for optimal mixture experimental design. Communications in Statistics-Theory and Methods. 2018; 47(6):1413-22. https://doi.org/10.1080/03610926.2017.1321122 